\title{
Toxic effects of monocrotophos on Paramecium caudatum
}

\author{
Ujwala Garad, Shanti N. Desai and Prakash V. Desai* \\ Department of Zoology, Goa University, Taleigao Plateau, Goa- 403521, India.
}

Accepted 13 August, 2007

\begin{abstract}
Many pesticides are used by agriculturist and monocrotophos (MCP) is one such pesticide used indiscriminately in India to control insect pests. MCP enters surface water and contaminates it. Paramecia have been used in the past for rapid toxicity assessment. Paramecia exposed to 1 to 100 ppm concentrations of MCP exhibit decline in population density, reduction of: overall body length, length and breadth of cytopharynx, rate of feeding and cyclosis. Technical grade MCP is toxic to Paramecium caudatum even at sub lethal concentrations.
\end{abstract}

Key words: Paramecium caudatum, monocrotophos, cytopharynx, feeding rate, cyclosis, pesticide.

\section{INTRODUCTION}

Water quality is essential for sustaining health of aquatic organisms and presence of toxicants in water poses threat to the organisms inhabiting such water bodies. The environmental protection agency report of 1990 suggests that pollution of rivers and ponds come mostly from agriculture (Cook et al., 1995). Many pesticides are used by agriculturists and monocrotophos (MCP) is one such organophosphate pesticide used indiscriminately in India to control insect pests. MCP is likely to enter surface water and cause water pollution, thereby affecting the organisms inhabiting it including paramecia. Paramecia have been used in the past for rapid toxicity assessment of pesticides (Komala, 1992; Juchelka and Snell, 1995; Rouabhi et al., 2006; Rao et al., 2007). The present study reports the effect of monocrotophos on the population density, body length, cytopharynx, feeding rate and cyclosis of Paramecium caudatum which is considered as an excellent cellular model for toxicity test (Rouabhi et al., 2006).

\section{MATERIALS AND METHODS}

One litre of pond water along with hydrophytes was collected from a pond behind the departmental building and water was examined microscopically for paramecia. Paramecium species was identified

*Corresponding author. E-mail: pvdesai@unigoa.ac.in. Tel: 91-832-2456133. as $P$. caudatum and was cultured by following the method of Wichterman (1952). Paramecia for experiments were selected from the log phase of cultures. MCP (technical grade, 95\% purity) was obtained from Ciba-Giegy, Goa and was dissolved in acetone to prepare a stock solution of $1 \mathrm{PPL}$. Then, with Sonneborn's medium (1970) the test solutions of 1, 10, 50 and 100 PPM strength were prepared. The exposure of paramecia to the various concentrations of MCP for 24, 48, 72, 96 and $120 \mathrm{~h}$ was carried out in $250 \mathrm{ml}$ glass beakers. Each beaker contained $100 \mathrm{ml}$ test concentration prepared in Sonneborn's medium and $10^{3}$ paramecia. Parallel controls were maintained in Sonneborn's medium. The kinetics of population growth was followed at the end of respective exposure period by determining the population density with the help of haemocytometer. The measurement of length of paramecium, length as well as breadth of cytopharynx, feeding rate and cyclosis was done using Media Cybernetics' Image- Pro Express Programme (USA). For determination of feeding rate in terms of ingestion of food particles, the control and test paramecia were introduced in the concavity slides (4 paramecia/concavity) and milk powder dyed with Congo red was added to each concavity. The paramecia were observed under microscope for 10 minutes and rate of ingestion of food particles/min was recorded. The experimental design for estimating ingestion rate and cyclosis in paramecium consisted of 15 replicates for each treatment and exposure period including the control. Along with the study of feeding rate, rate of cyclosis, the rotary streaming movement of endoplasm which carries the food vacuole along a definite course was determined in terms of time taken for completion of one cycle.

\section{RESULTS AND DISCUSSION}

Monocrotophos at 1 and $10 \mathrm{ppm}$ concentrations did not affect the population density of paramecia exposed to it 


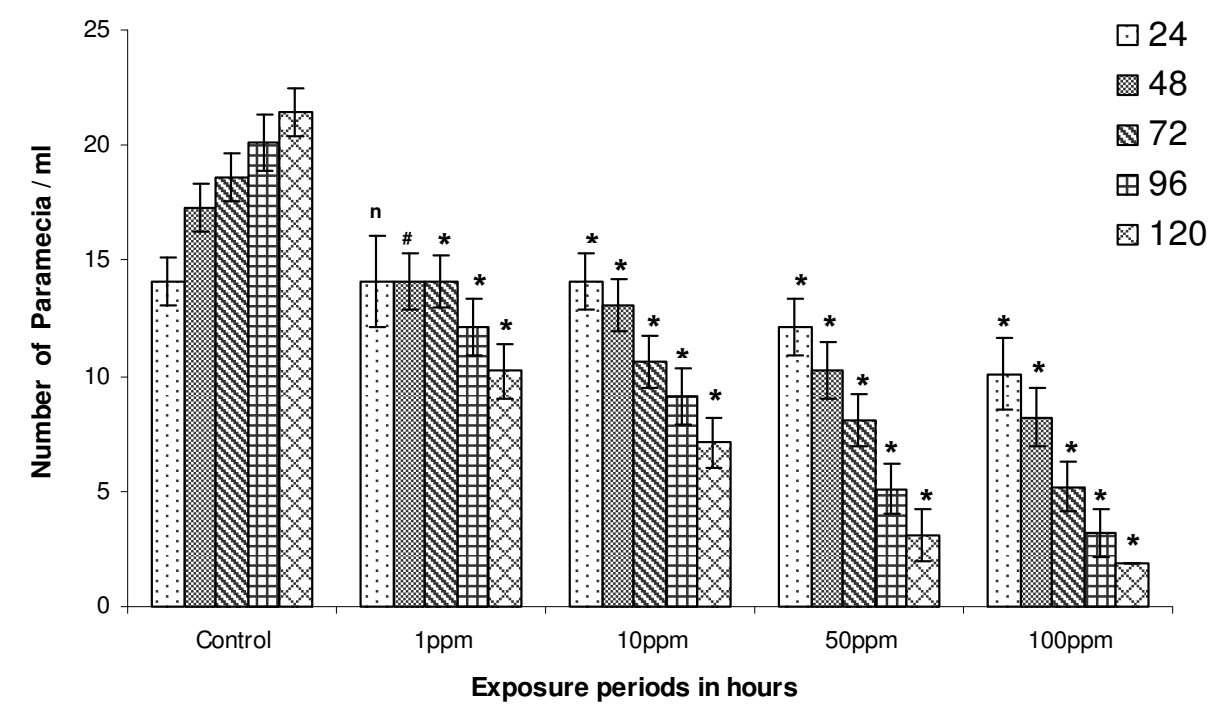

Figure 1. Effect of monocrotophos exposure on population density count of Paramecium caudatum. Data are mean $\pm \mathrm{SD}$ for ten independent experiments. ${ }^{*}$ Different from control, $\mathrm{P}<$ 0.001 ; \# $\mathrm{P}<0.01 ; \mathrm{n}$, insignificant (Student's t-test).

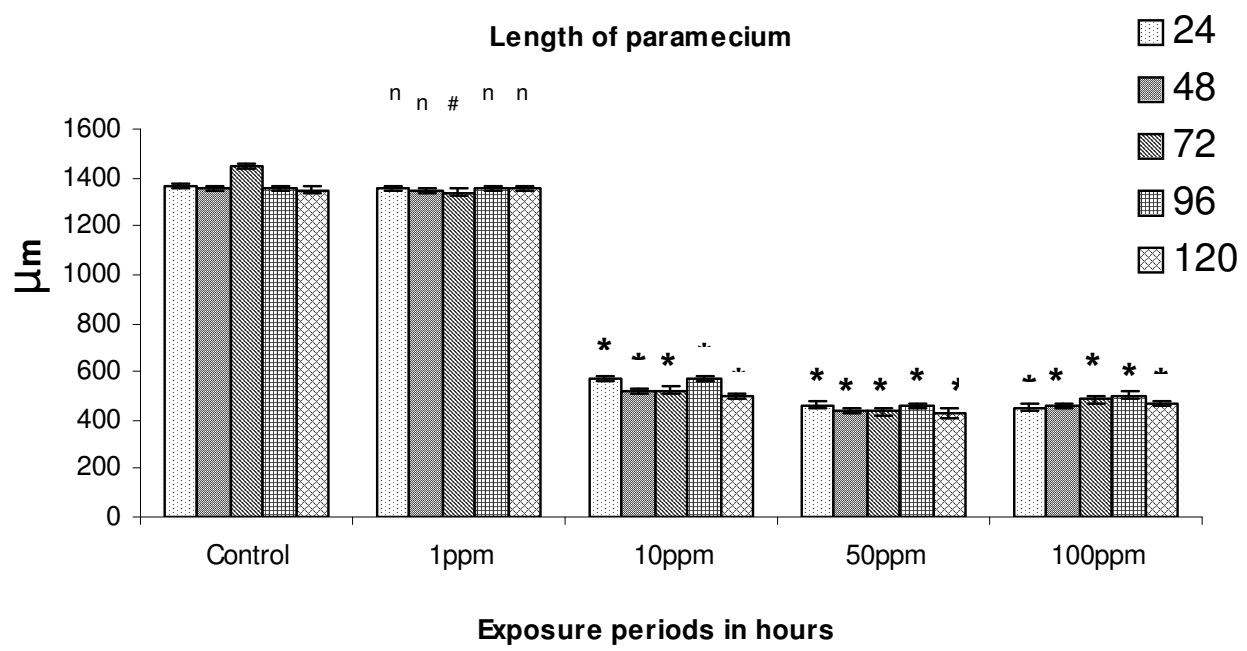

Figure 2. Effect of monocrotophos exposure on length of Paramecium caudatum. Data are mean \pm SD for ten independent experiments performed ten times. ${ }^{*}$ Different from control, $\mathrm{P}<$ $0.001 ; \# \mathrm{P}<0.01 ; \mathrm{n}$, insignificant (Student's t-test).

for $24 \mathrm{~h}$ but exposures for a period longer than $24 \mathrm{~h}$ promoted significant decrease in population density of paramecia at all the test concentrations (Figure 1). The length of paramecia was reduced prominently by a concentration of monocrotophos above $1 \mathrm{ppm}$ at exposure periods of 48 to $120 \mathrm{~h}$ (Figure 2) but the degree of decrements in breadth and length of cytopharynx was dependent upon the test concentration of monocrotophos (Figures 3 and 4). Similarly, the feeding and cyclosis rates of paramecia declined in a dose dependent manner (Figures 5 and 6). Here the increase in the time taken for completion of cyclosis of food vacuoles under the influence of monocrotophos is in reality a decrease in rate of cyclosis. The morphological studies showed the changes in shape and size of paramecia (Figures 7 and 8).

Contribution of microorganisms to the primary productivity, nutrient cycling in aquatic ecosystem is well understood. Therefore, contamination of aquatic ecosystem with pesticides would have detrimental effects on micro fauna. The pesticides reach aquatic ecosystem through spills, runoff and these pesticides are reported to affect the structure and function of microorganisms like paramecia (Edmiston et al., 1985; Rajini et al., 1989; Komala, 1985, 1992; Juchelka and Snell, 1995; Galal, 1996; Norikazu et al., 2003; Rouabhi et al., 2006; Rao et al., 


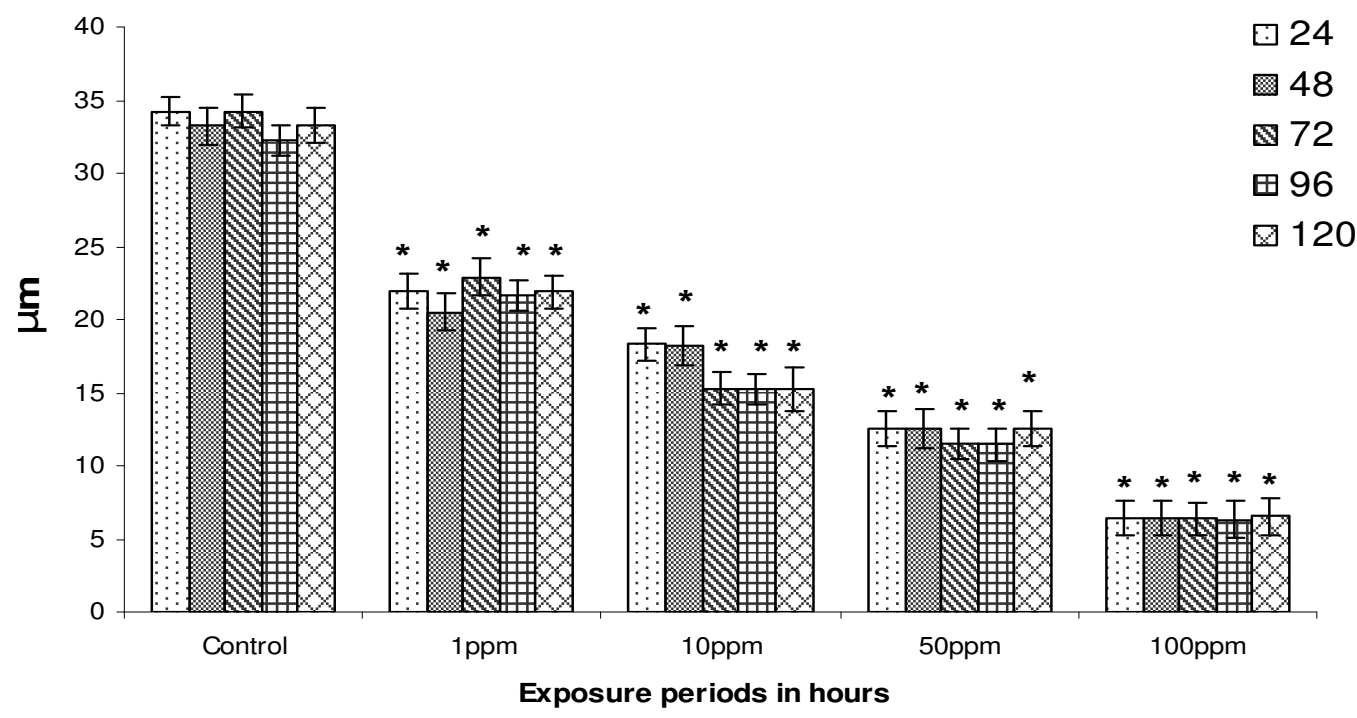

Figure 3. Effect of monocrotophos exposure on length of cytopharynx of Paramecium caudatum. Data are mean \pm SD for ten independent experiments performed ten times. *Different from control, $\mathrm{P}<0.001$ (Student's t-test).

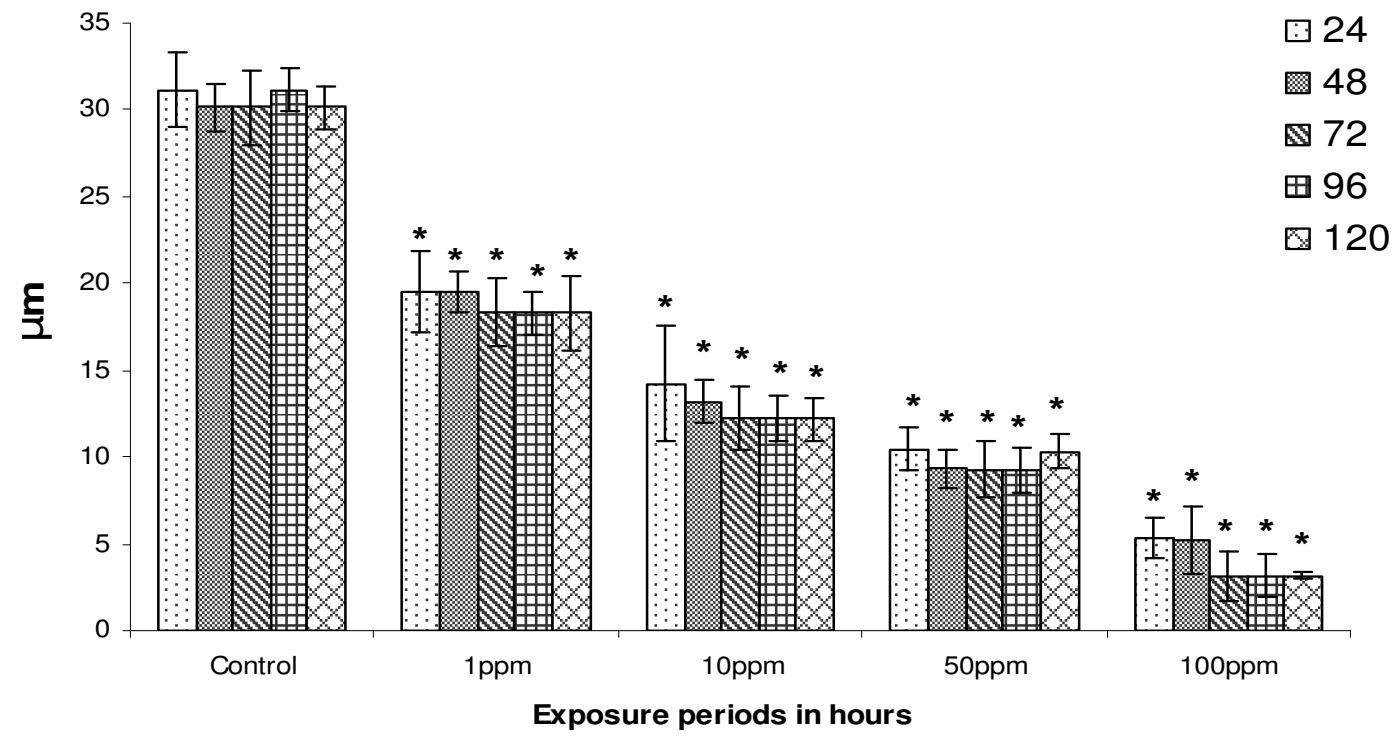

Figure 4. Effect of monocrotophos exposure on breadth of cytopharynx of Paramecium caudatum. Data are mean \pm SD for ten independent experiments performed ten times. *Different from control, $\mathrm{P}<0.001$ (Student's t-test).

2007). Rao et al. (2007) exposed Paramecium caudatum to different sub lethal concentrations $(5,10,15$ and 20.0 $\mathrm{mg} \mathrm{L}^{-1}$ ) of monocrotophos and observed that number of generations of paramecia decreased with extension of generation time in a concentration dependent manner. Further, they determined lethal concentrations (50\%) by probit method for technical grade monocrotophos as 60 and $40.6 \mathrm{mg} \mathrm{L}^{-1}$ in static toxicity tests of $10 \mathrm{~min}$ and $24 \mathrm{~h}$ respectively. In the present investigation, we have used sub lethal concentrations at a very low level $(1,10,50$ and $100 \mathrm{ppm}$ ) than reported by Rao et al. (2007) and found that monocrotophos is toxic to Paramecium caudatum with reference to decline in population. This population decline is partly due to death of paramecia as observed by us and may partly be due to decrease in generations due to failure of asexual and sexual reproduction coupled with extension of generation time.

The reduction of length of paramecia under the influence of monocrotophos appears to be partly due to a longitudinal contraction of the body and partly due to the 


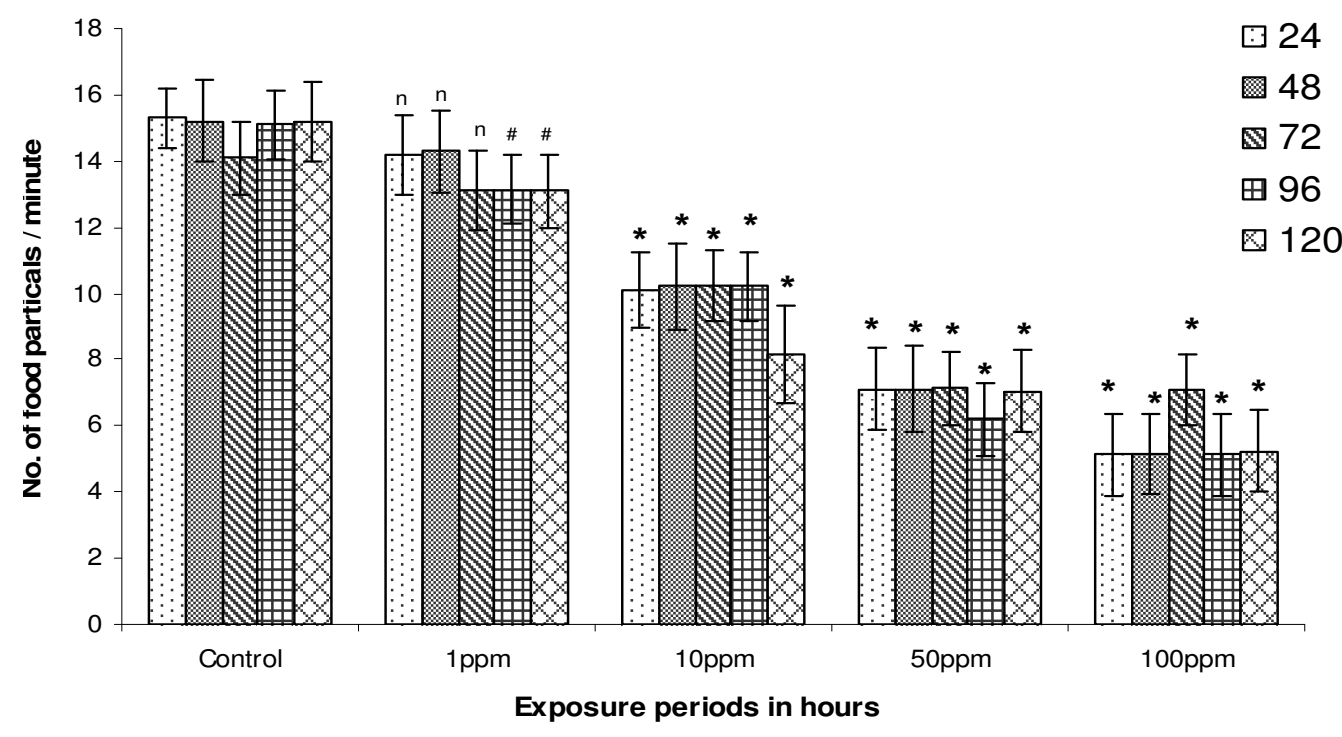

Figure 5. Effect of monocrotophos exposure on feeding rate in Paramecium caudatum. Data are mean $\pm S D$ for ten independent experiments performed ten times. *Different from control, $P<0.001$; \# $\mathrm{P}<0.01 ; \mathrm{n}$, insignificant (Student's t-test).

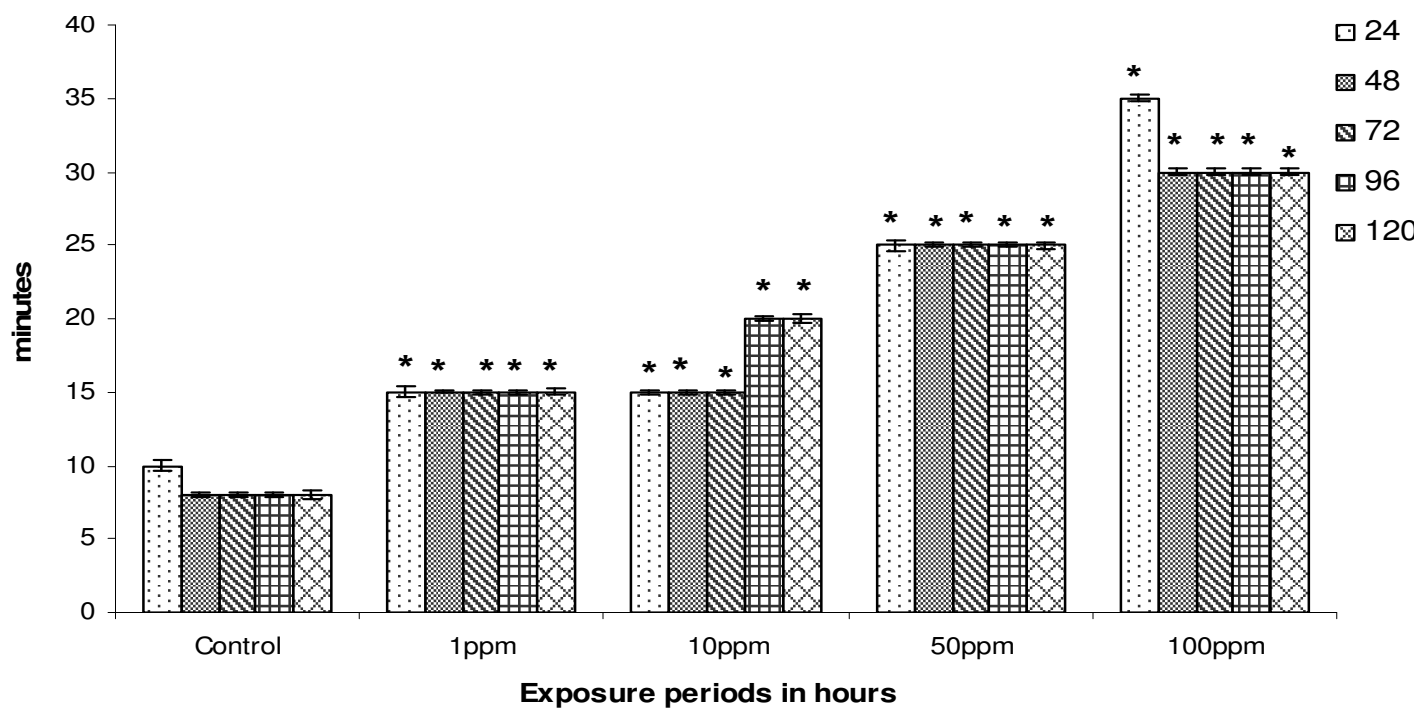

Figure 6. Effect of monocrotophos exposure on rate of cyclosis of food vacuole in Paramecium caudatum. Data are mean $\pm S D$ for ten independent experiments performed ten times. ${ }^{*}$ Different from control, $P<0.001$ (Student's t-test).

loss of cytoplasm resulting into redistribution of remaining cytoplasm for promoting cyclosis, an event associated with digestion. The reduction of length and breadth of cytopharynx under the influence of all concentrations of monocrotophos could be due to contraction of the contractile elements of cytopharynx. The reduction of the feeding rate could be due to the reduction of breadth and length of cytopharynx which is associated with ingestion of food particles vis-à-vis formation of food vacuoles, particularly the reduction of breadth of cytopharynx could have promoted narrowing of the passage of food particles vis-à-vis impaired the process of formation of food vacuoles. Kooijman and Metz (1984) are of the opinion that toxicant induced reductions of food ingestion rates may lead to reduced survival and adverse consequences at population level. Juchelka and Snell (1995) observed reduction of food particles ingestion in Paramecium aurelia exposed to pentachlorophenol and chlorpyrifos.

The increase in time taken by paramecia to complete one cycle of food vacuole movement that is, cyclosis indicates reduced streaming of cytoplasm essential for onward movement of food vacuoles for completion of 


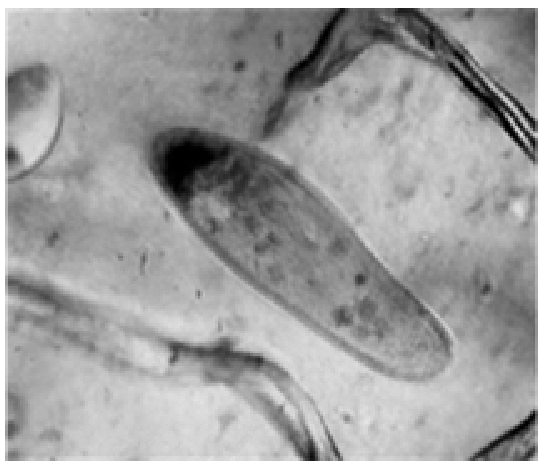

A

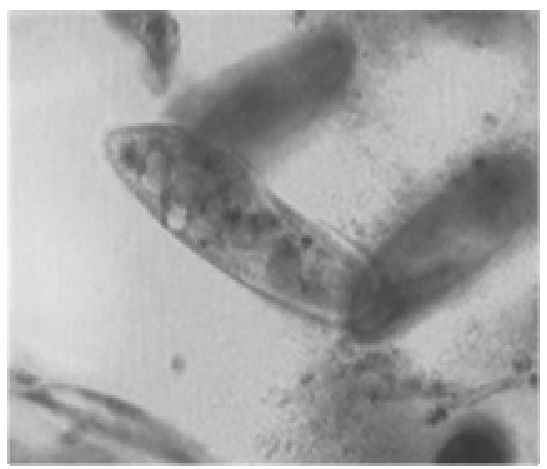

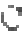

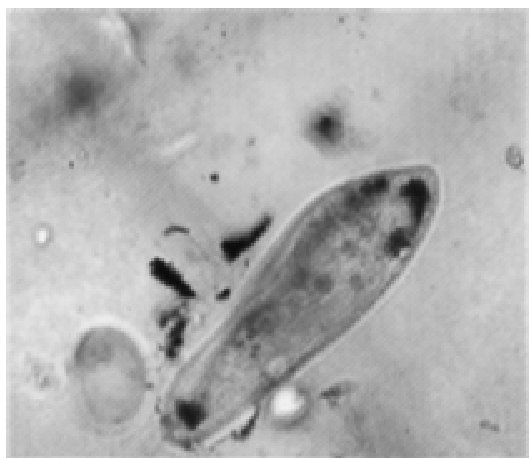

E

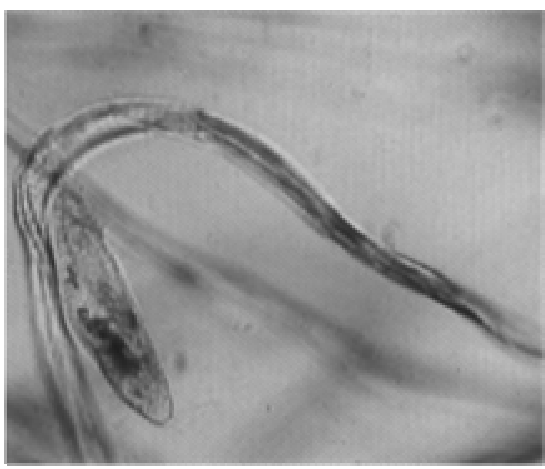

D

Figure 7. (10x) Morphological changes in Paramecium: A, Control showing normal shape and size; $B$, under the influence of $1 \mathrm{ppm} ; C$ and $D$ under the influence of 50 ppm and 100 ppm showing reduction in size.

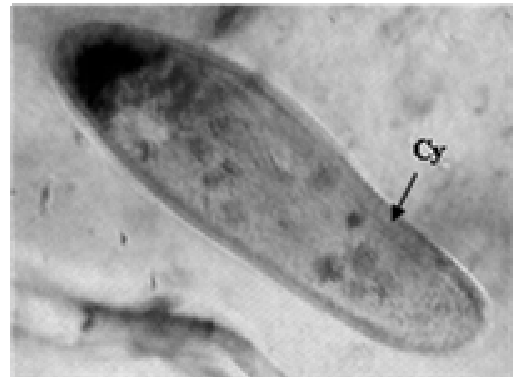

A

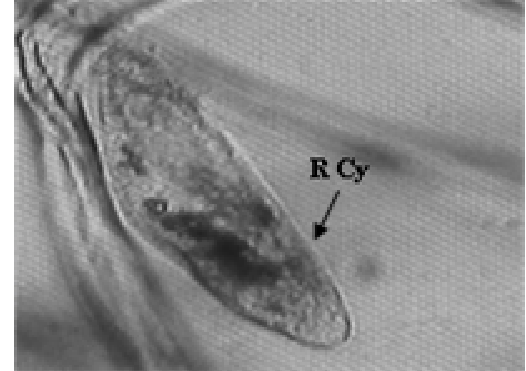

B

Figure 8. (20x) A, Control showing normal shape and size cytopharynx (Cy) and $B$, showing reduced cytopharynx ( $\mathbf{R} \mathbf{C y}$ ) and general cell structure of paramecium under the influence of $100 \mathrm{ppm}$ monocrotophos.

digestion. The reduced length and volume of paramecia coupled with loss of cytoplasm could have impaired cytoplasmic streaming. The reduction of the rate of cyclosis would delay or impair digestion of food thereby reducing the energy budget of the animal. Rao et al. (2007) have reported decrease in mobility as well as locomotory behaviour of Paramecium caudatum under the influence of high concentration of monocrotophos. This behavioural change could be due to reduced energy budget promoted by reduced ingestion rate and cyclosis as observed in the present investigation. Thus, it appears that even a low concentration of monocrotophos promo- 
tes reduction of population density, length of animal as well as reduction of length and breadth of cytopharynx which leads to reduction of feeding rate as well as cyclosis.

\section{REFERENCES}

Cook JL, Bauman P, Jackman JA, Stevenson D (1995). Pesticide Characteristics that affect water quality. Farm Chemical Handbook, 95. Meister Publishing Co. Willoughby OH. p. 429.

Edmiston Jr CE, Goheen M, Malaney GW, Mills WL (1985). Evaluation of Carbamate toxicity: Acute toxicity in a culture of Paramecium multimicronucleatum upon exposure to aldicarb, carbaryl, inmexacarbate as measured by Warburg respirometry and acute plate assay. Environ. Res. 36(2): 338-350.

Galal M (1996). Experimental studies on the effect of some pesticides on the densities and the growth rates of particular bacteriovorous ciliates. J. Egypt. Ger. Soc. Zool. 20: 27-42.

Juchelka CM, Snell TW (1995). Rapid toxicity assessment using ingestion rate of Cladocerans and Ciliates. Arch. Environ. Contam. Toxicol. 28: 508-512.

Komala Z (1992). Toxicity of Fastac 10 EC, a pyrethroid insecticide to Paramecium primaurelia and Tubifex sp. Folia Biol (Krakow). 40(3-4): 109-112.
Komala Z (1995). Notes on the use of invertebrates, especially ciliates, in studies on pollution and toxicity. Folia Biol (Krakow) 43: 25-27.

Kooijman S, Metz JAJ (1984). On the dynamics of chemically stressed Populations: The deduction of population consequences from individual effects. Ecotox. Environ. Safety. 8: 254-274.

Norikazu M, Tomonori K, Miho T, Takashi K, Toshikazu K, Manadu K, Tadao T, Hiroshi H (2003). Use of paramecium species in bioassay for environmental risk management: Determination of IC50 values for water pollutants. J. Health. Sci. 49: 429- 435.

Rajini PS, Krishnakumari MK, Mujumder SK (1989). Cytotoxicity of certain Organic solvents and organophosphorus insecticides to the ciliated protozoan Paramecium caudatum. Microbios 59: 157-163.

Rao VJ, Gunda VG, Srikanth K, Arepalli SK (2007). Acute toxicity bioassay using Paramecium caudatum, a key member to study the effects of monocrotophos on swimming behaviour, morphology and reproduction. Toxicol. Environ. 89(1) DOl-10 1080102772240601010071.

Rouabhi R, Berrebbah H, jebar MR (2006). Toxicity evaluation of Flucycloxuron and diflubenzuron on the cellular model, paramecium sp. Afr.J. Biotechnol. 5(1): 45-48.

Sonneborn TM (1970). Paramecia. In Prescot D.M. (Ed) Methods in Cell Physiology. Vol.4, Academic Press New York, p. 241.

Wichterman R (1952). A method for obtaining abundant dividing stages of Paramecium. Trans. Am. Microsc. Soc. 71(3): 303-305. 